\title{
COVID-19 pansinusitis with abducens paralysis
}

\author{
Meral Seferoğlu${ }^{1}$, Nizameddin Koca ${ }^{2} \odot$, Yasemin Çoşkun ${ }^{3} \odot$, Bahattin Hakyemez ${ }^{4}$ \\ ${ }^{1}$ Department of Neurology, University of Health Sciences, Bursa Yüksek Ihtisas Training and Research Hospital, Bursa, Turkey \\ ${ }^{2}$ Department of Internal Medicine, University of Health Sciences, Bursa Yüksek İhtisas Training and Research Hospital, Bursa, Turkey \\ ${ }^{3}$ Department of Infectious Disease, University of Health Sciences, Bursa Yüksek Ihtisas Training and Research Hospital, Bursa, Turkey \\ ${ }^{4}$ Department of Radiology, Bursa Uludă̆ University School of Medicine,, Bursa, Turkey
}

\begin{abstract}
Increasing numbers of the papers indicate that SARS-CoV-2 also causes neurological symptoms; the underlying mechanism has not been elucidated yet. Hypothetic mechanisms to explain the CNS involvement of SARS$\mathrm{CoV}-2$ include the neurotropic mechanisms and the cytokine storm developing during the disease process. A middle age female patient applied to the emergency department with complaints of eye pain, a double, foggy, and blurred vision and a severe throbbing headache. The outward gaze was found to be limited in her right eye. Nasopharyngeal swab for SARS-CoV-2 RNA was positive, radiological findings were supported the COVID pneumonia and diffuse sinonasal inflammation. Cranial imaging showed thickening and contrast involvement in the cavernous sinus in the postcontrast series. While shortness of breath improved, and the headache was completely resolved on the 10th day of treatment the right eye outward gaze restriction was continued. The control MRI reveals a significant reduction in cavernous thickening and contrast enhancement and complete resolution in dural thickening. In our case of COVID, cranial nerve involvement and pansinusitis developed without cytokine storm findings suggests that the virus has spread to the cavernous sinuses and dura by regional neighborhood. Neurological symptoms may appear as the first symptom of COVID.
\end{abstract}

Keywords: COVID-19, pansinusitis, abducens paralysis, headache

$\mathbf{M}$ iddle East Respiratory Syndrome (MERS-CoV) and Severe Acute Respiratory Syndrome (SARS-CoV-1) viruses have been shown to cause brain damage. Although increasing numbers of the papers indicate that SARS-CoV-2 also causes neurological symptoms, the underlying mechanism has not been elucidated yet. Hypothetic mechanisms to explain the CNS involvement of SARS-CoV-2 include the neurotropic mechanisms and the cytokine storm developing during the disease process [1]. The passage of the virus into the brain tissue can be by crossing the blood brain barrier(BBB) or by transsynaptic passage from infected neurons through the olfactory nerve [1]. SARS-CoV-2 has 10 to 20 times more affinity to
ACE2 than SARS-CoV-1. Systemic inflammation that develops during the course of COVID-19 infection probably increases the permeability of BBB, allows infected immune cells, cytokines, and possibly the virus itself to pass to CNS [2].

In this report, we shared a COVID-19 case with the $6^{\text {th }}$ cranial nerve palsy accompanied by pansinusitis in which neurological symptoms were the initial findings.

\section{CASE PRESENTATION}

A 60-years-old female with no prior history of chronic disease and drug use, applied to the emer- 
gency department with the complaints of ocular pain, double, foggy and blurred vision which had started after severe throbbing headache. She also had nausea, postnasal drip, accompanying mucus sputum, anosmia and ageusia. She was hospitalized and hydroxychloroquine $(2 \times 200 \mathrm{mg})$, azithromycin $(500 \mathrm{mg} 1 \times 1)$, and low molecular weight heparin (Enoxaparin 6000 anti-

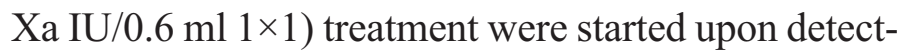
ing the findings supporting the COVID pneumonia (Fig. 1. A1 and A2) on the thorax computed tomography (CT). Severe loss of ventilation and diffuse sinonasal inflammation was observed in the paranasal sinus CT (Fig. 1B).

Her nasopharyngeal swab for SARS-CoV-2-RNA was positive. Green and foul-smelling diarrhea was added to her complaints on the 5th day of hospitalization. No fever observed. She was conscious, cooperated and orientated. Her visual acuity allows her to count fingers from 6 meters with both eyes. There were full muscle strenght on both upper and lower extremities, deep tendon reflexes were normoactive, sen- sory, and cerebellar tests were within normal limits. Muscle strength fluctuations was not observed during the follow-up. There was no ataxia and the Romberg test was negative. Fundus examination was normal and there was no evidence of papillary edema. The outward gaze was found to be limited in her right eye.

Magnetic resonance imaging (MRI) of the brain showed widespread contrast inflammation in the paranasal sinuses, as well as thickening and contrast involvement in the cavernous sinus in the postcontrast series. Diffuse thickening was observed in the posterior parietal and occipital wall (Fig. 1C and 1D). No sign of venous sinus thrombosis was observed.

The cerebrospinal fluid (CSF) examination revealed an opening pressure of $11 \mathrm{cmH} 2 \mathrm{O}$ (reference values $7-20 \mathrm{cmH} 2 \mathrm{O}$ ), protein $=26.75 \mathrm{mg} / \mathrm{dL}$ (reference values: $15-45 \mathrm{mg} / \mathrm{dL}$ ), glucose $=59 \mathrm{mg} / \mathrm{dL}$ (reference values: $40-70 \mathrm{mg} / \mathrm{dL}$ ) with normal cytology. The bacterial culture, serological tests, routine blood tests and SARS-CoV-2 PCR of CSF was negative. Antibiotics and Favipiravir treatment was added to for-

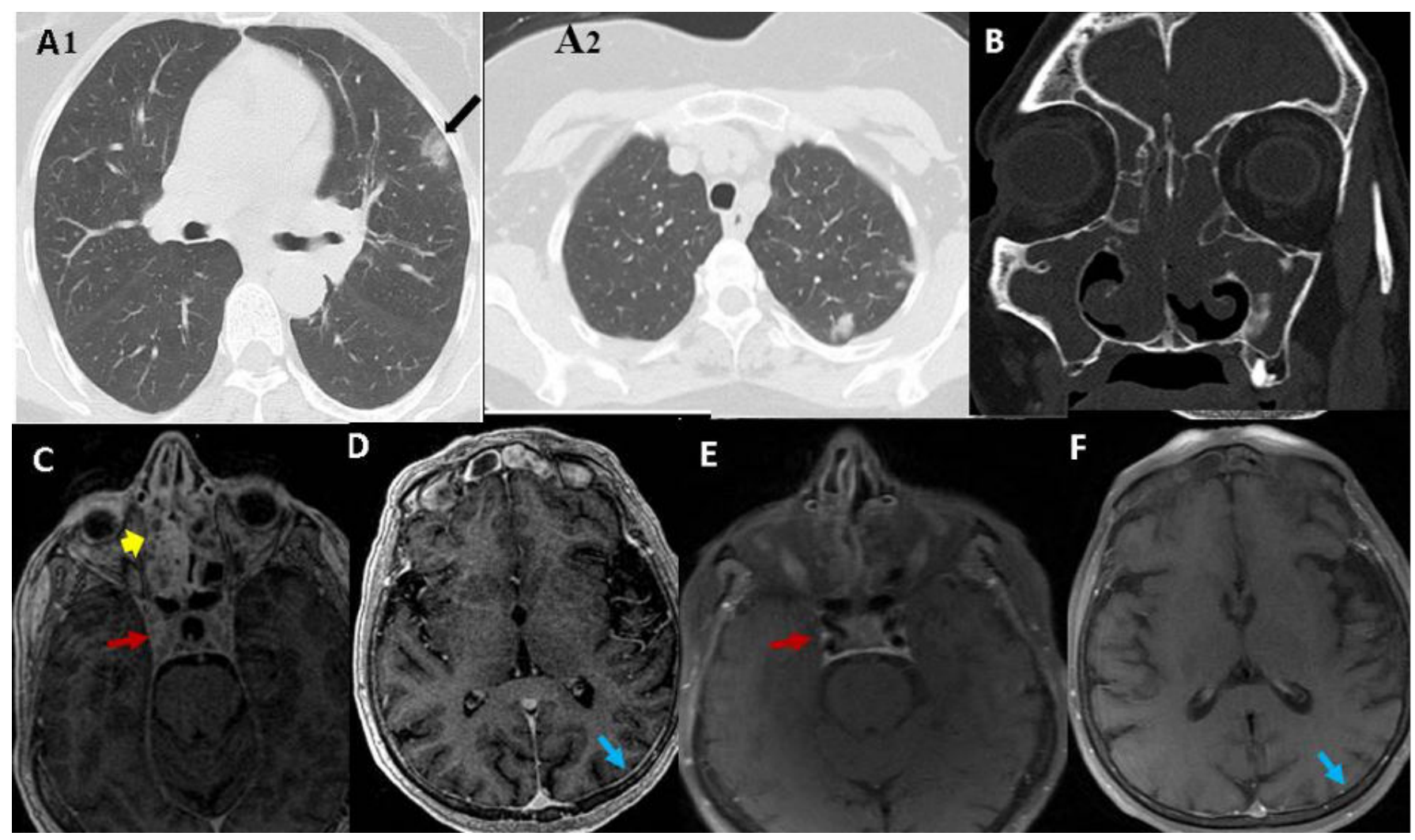

Fig. 1. (A1 and A2) = Parenchymal infiltration area (black arrow) in the left lung, upper lobe anterior-lateral, ground-glass density. (B) = Widespread inflammation and loss of aeration in the paranasal sinuses. (C-D) = Cavernosal thickening (red arrow) and sinonasal inflammation (yellow arrow) and diffuse thickening in the posterior parietal wall (blue arrow) in the postcontrast MR before treatment. (E-F) = Decline in cavernosal (red arrow) and dural thickening (blue arrow) in postcontrast MR after treatment. 
mer treatment. Except right outward gaze restriction, headache was completely resolved, and shortness of breath improved on the $10^{\text {th }}$ day of treatment. Cavernous thickening and contrast enhancement were significantly reduced, and dural thickening was completely resolved in the control cranial MRI (Fig. $1 \mathrm{E}$ and $1 \mathrm{~F})$.

\section{DISCUSSION}

The smell and taste disturbances commonly described in the course of various viral infections. These symptoms are reported to be seen independently of respiratory signs and symptoms during the COVID19 disease process. Although these symptoms are not specific for COVID-19 they suggest olfactory bulb involvement, which is thought to be a way for the viruses to enter into the central nervous system [3]. In animal models, it has been shown that SARS-CoV-1, and MERS-CoV can enter to the brain through the olfactory nerves and then spread rapidly to certain brain regions [2]. In our case of COVID-19, cranial nerve involvement and pansinusitis developed without cytokine storm findings suggesting that the virus has spread to the cavernous sinuses and dura by local transmission. Marc Dinkin et al. [4] reported a case of abducens nerve involvement without radiological evidence and stated that an increase in the optic nerve sheath of the included eye may reflect viral leptomeningeal invasion. In our case, the fact that anosmia and ageusia are among the findings accompanying pansinusitis, as well as the presence of contrast enhancement on the side where there is abducens paralysis radiologically, seems to support this theory.

Myasthenia gravis, Guillain-Barre and MillerMisher syndromes should be considered in differential diagnosis. Cranial nerve involvement is mostly associated with Guillain-Barre or Miller-Fisher syndromes which thought a parainfectious or postinfectious conditions [5]. Anamnesis and physical examination findings during the follow-up did not suggest these diagnoses. Unlike the others, neurological findings such as severe headache and cranial nerve involvement were observed as symptoms of presentation in our case. Response to the treatment was objectively documented with detailed neurological examination and cranial MRI results before and after treatment.

\section{CONCLUSION}

Neurological symptoms may appear as the first symptom of COVID-19. Detailed neurological examination, CSF analysis, and MRI are very important in depicting the course and spread of the disease. The neuroinvasive and neurotropic features of the SARS$\mathrm{CoV}-2$ virus may have neuropathological effects under unpredictable environmental and background genetic factors. This is a case report with evidence supporting the neuroinvasion of SARS-CoV-2 to the brain.

\section{Authors' Contribution}

Study Conception: MS, NK, YÇ, BH; Study Design: MS, NK Supervision: MS, NK; Funding: N/A; Materials: N/A; Data Collection and/or Processing: MS, YÇ; Statistical Analysis and/or Data Interpretation: MS, NK, YÇ, BH; Literature Review: MS, NK; Manuscript Preparation: MS, NK and Critical Review: MS, NK, BH.

\section{Ethical statement}

The case report was written with the guidance of CARE guideline

\section{Conflict of interest}

The authors disclosed no conflict of interest during the preparation or publication of this manuscript.

\section{Informed consent}

Written informed consent was obtained from the patient for publication of this case and any accompanying images.

\section{REFERENCES}

1. Vonck K, Garrez I, De Herdt V, Hemelsoet D, Laureys G, Raedt R, et al. Neurological manifestations and neuro-invasive mechanisms of the severe acute respiratory syndrome coronavirus type 2. Eur J Neurol 2020;27;1578-87.

2. Li Z, Liu T, Yang N, Han D, Mi X, Li Y, et al. Neurological manifestations of patients with COVID-19: potential routes of SARS-CoV-2 neuroinvasion from the periphery to the brain. Front Med 2020;14;533-41.

3. Zubair AS, McAlpine LS, Gardin T, Farhadian S, Kuruvilla DE, Spudich S. Neuropathogenesis and neurologic manifestations of the coronaviruses in the age of coronavirus disease 2019: a review. JAMA Neurol 2020;77:1018-27.

4. Dinkin M, Gao V, Kahan J, Bobker S, Simonetto M, Wechsler 
P, et al. COVID-19 presenting with ophthalmoparesis from cranial nerve palsy. Neurology 2020;95:221-3.

5. Gutiérrez-Ortiz C, Méndez A, Rodrigo-Rey S, San Pedro-
Murillo E, Bermejo-Guerrero L, Gordo-Mañas R, et al. Miller Fisher Syndrome and polyneuritis cranialis in COVID-19. Neurology 2020;95:e601-5. 